\title{
La restauration des zones humides
}

\author{
J.P. Berton, M. Bacchi \\ Université de Tours (IMACOF)
}

La notion de «zone humide» a été introduite à la fin des années 1960 par les scientifiques et les protecteurs de la nature préoccupés par le devenir de ces milieux temporaires menacés. Dans le cadre du projet MAR 1, première opération internationale s'intéressant à la conservation des zones humides, Jouanin [1] notait que ce terme «désigne des milieux biologiques que nous appelons selon les cas, marais, marécage, vasière, estuaire, lagune, étang, etc. C'est une désignation générale qui englobe des milieux variés, mais ayant tous entre eux deux caractères communs: la présence de l'eau et la faible profondeur de celle-ci.

\section{DEFINITION, TYPOLOGIE ET FONC- TIONNEMENT DES ZONES HUMIDES}

\subsection{Définition des zones humides}

Caractériser les zones humides reste difficile, aucune définition scientifique ne faisant l'unanimité à l'échelle internationale [2]; par ailleurs, l'expert, selon sa spécialité (géologie, pédologie, hydrologie, géographie, biologie, écologie, sociologie, économie, droit, urbanisme) a sa propre définition adaptée à ses propres objectifs.

Pourtant, trois raisons principales rendent nécessaire des définitions précises :

- l'avènement des législations environnementales nationales, européennes, oblige à identifier précisément les objets visés par les réglementations, ces dernières ayant des conséquences foncières et fiscales importantes;

- on doit disposer de données quantifiées, de cartes et généralement d'arguments pertinents pour sauvegarder les zones humides face aux projets d'aménagement, aux risques de pollution, ...

- la gestion de la ressource en eau (qualité, quantité), implique d'identifier les rôles joués par les zones humides vis-à-vis de cette ressource pour envisager un raisonnement global et durable [3].

\subsubsection{Aspect juridique}

Deux définitions existent dans la législation française :

- A l'échelle internationale, dans le cadre de la convention de Ramsar (1971), ratifiée par la France, relative aux zones humides d'importance internationale en ce qui concerne les habitats des oiseaux d'eau, on trouve la définition suivante :
«Les zones humides sont des étendues de marais, de fagne, de tourbières ou d'eaux naturelles ou artificielles, permanentes ou temporaires, où l'eau est stagnante ou courante, douce, saumâtre ou salée, y compris des étendues d'eau marine dont la profondeur à marée basse n'excède pas six mètres.» Il est en outre précisé que les zones humides pourront inclure des zones de rives ou de côtes adjacentes à la zone humide et des îles ou étendues d'eau marine d'une profondeur supérieure à six mètres à marée basse, entourées par la zone humide.» (J.O. du 26/02/1987)

- A l'échelle nationale, la loi sur l'eau du 3 jànvier 1992 donne la définition suivante :

«Art. 2.- Les dispositions de la présente loi ont pour objet une gestion équilibrée de la ressource en eau. Cette gestion équilibrée vise à assurer la préservation des écosystèmes aquatiques, des sites et des zones humides; on entend par zones humides les terrains, exploités ou non, habituellement inondés ou gorgés d'eau douce, salée ou saumâtre de façon permanente ou temporaire; la végétation, quand elle existe, y est dominée par des plantes hygrophiles pendant au moins une partie de l'année».

\subsubsection{Aspect scientifique/écologique}

Le biologiste regrettera cependant que ni l'une ni l'autre de ces définitions ne soulignent que ces zones constituent des espaces de transition entre la terre ferme et l'eau libre généralement soumises à un gradient d'humidité et qu'à ce titre, elles se comportent comme des lisières.

On peut les qualifier d'interface, d'écosystèmes de transition souvent sans frontières nettes car en position de lisière dans l'espace et le temps. Certains peuvent être considérés comme des écotones : zone de transition entre des systèmes écologiques adjacents possédant un ensemble de caractéristiques définies uniquement par des échelles spatiales et temporelles et par la force des interactions des écosystèmes adjacents. (groupe de travail IUBS-Unesco MAB-SCOPE [4] )

Une consultation d'experts français a permis d'aboutir (1990) à la définition suivante:

«Les zones humides se caractérisent par la présence, permanente ou temporaire en surface ou à faible profondeur dans le sol, d'eau disponible, douce, saumâtre ou salée souvent en position d'interface, de transition entre milieux terrestres et milieux aquatiques proprement dits. Elles se distinguent par une faible profondeur d'eau, des sols 
hydromorphes ou non évolués, et/ou une végétation dominante composée de plantes hygrophiles au moins pendant une partie de l'année. Enfin, elles nourrissent et/ou abritent de façon continue ou momentanée des espèces animales inféodées à ces espaces.

Les zones humides correspondent aux marais, marécages, fondrières, fagnes, pannes, roselières, tourbières, prairies humides, marais agricoles, landes et bois marécageux, forêts alluviales et ripisylves marécageuses, mares y compris les temporaires, étangs, bras morts, grèves à émersion saisonnière, vasières, lagunes, prés salés, marais salicoles, sansouires, rizières, mangroves, etc.

Elles se trouvent en lisière de sources, de ruisseaux, de fleuves, de lacs en bordure de mer, de baies et d'estuaires, dans les deltas, dans les dépressions de vallée ou dans les zones de suintement à flanc de collines.» [5].

\subsection{Typologies des zones humides}

La typologie est la science de l'élaboration des types, qui facilite l'analyse d'une réalité complexe et la classification. C'est un découpage du réel en différentes unités, rassemblées ensuite dans des catégories représentant chacune un type. En ce qui concerne les milieux naturels, nous sommes en présence d'«objets» qui évoluent dans le temps et cet aspect dynamique complique la tâche.

Il existe de nombreuses classifications des milieux naturels pour l'ensemble du globe et pour les zones humides, une multiplicité de systèmes généraux ou thématiques applicables à l'échelle mondiale, nationale, régionale, locale.

Exemple international : convention de Ramsar (1971).

Inventaire des zones humides d'importance internationale particulièrement comme habitat des oiseaux d'eau.

Exemples européens : CORINE-Land cover (1989)

Cartographie des écozones ou «unités d'analyse paysagère» homogènes reposant sur les caractéristiques des territoires étudiés à partir des images de la télédétection. Parmi les zones humides une distinction est faite entre celles de l'intérieur et les côtières.

\section{CORINE-Biotopes (1991)}

Il s'agit d'un inventaire de sites d'importance communautaire et désignation des Zones Spéciales de Conservation (ZSC) dans le cadre de l'application de la directive «Habitat». La typologie se fonde sur des caractéristiques de la végétation. Huit grandes catégories ont été définies: communautés côtières et halophiles, eaux non marines, landes et pelouses, forêts, tourbières et marais, habitats rocheux, éboulis et sables, terrains agricoles et paysages artificialisés; de cette typologie on peut extraire les éléments concernant les zones humides.

\section{Exemples français}

- Les typologies des espaces naturels [6, 7].

Il s'agit d'une typologie réalisée pour la cartographie de l'Inventaire Permanent du Littoral.

En ce qui concerne les zones humides:
- Zone Naturelle d'Intérêt Écologique, Faunistique, Floristique (ZNIEFF, 1982)

Il s'agit d'un inventaire national des milieux naturels. Treize types sont localisés en zones marines et côtières (mer ouverte à proximité d'une côte, baie-détroit, estuaire, delta, côte rocheuse, falaise, plage, dune, île, lagune-lac et étangs d'eau salée (côtiers), lac, étang et marais d'eau douce, marais salant, vasière (côtière), pré salé) et six en zones humides intérieures (cours d'eau rapide, cours d'eau lent, lac-réservoir-étang, marais-tourbière, prairie humide, bassin salé) et un type en haute mer.

En 1993, environ $29000 \mathrm{~km}^{2}$ de zones ZNIEFF à dominante zone humide ont été recensées, soit environ $5 \%$ du territoire.

L'actualisation en cours de l'inventaire ZNIEFF utiliserait la typologie CORINE-Biotopes [8].

- Observatoire des zones humides [9].

Il s'agit de l'inventaire des zones humides d'importance majeure au plan national.

- Évaluation des politiques publiques de protection, de gestion et d'aménagement des zones humides [10].

Il s'agit d'une proposition de typologie basée sur une combinaison des caractéristiques écologiques des milieux en jeu et des ensembles de politiques publiques impliquées dans leur évolution. 12 types sont identifiés comprenant 87 sites qui sont essentiellement ceux inventoriés par l'Observatoire des zones humides:

- zones humides littorales : baies rocheuses, baies et estuaires moyens plats, grands estuaires, marais et lagunes côtiers, marais saumâtres aménagés.

- zones humides intérieures : zones humides de montagnes, collines et plateaux, marais agricoles aménagés, régions d'étangs, plaines humides mixtes liées aux cours d'eau, zones humides liées à un plan d'eau ponctuel ;

- zones humides des cours d'eau et bordures boisées marais et landes humides de plaine.

- SDAGE - SAGE [11]

La typologie précédente a été adaptée aux besoins des Agences de l'Eau. L'application de la loi sur l'eau, pour une gestion qualitative et quantitative de la ressource, implique la mise en oeuvre de Schémas Directeurs d'Aménagement et de Gestion des Eaux (SDAGE) par grand bassin et de Schéma d'Aménagement et de Gestion des Eaux par unité hydrographique cohérente (SAGE).

Cette typologie intègre les notions de fonction et services. rendus par les zones humides considérées alors comme des infrastructures naturelles (voir plus loin).

Cette typologie comprend des niveaux emboîtés pour les SDAGE-SAGE et tient compte des facteurs dominants, liés aux caractéristiques chimiques de l'eau (salée, saumâtre, douce) et au régime hydrique (eau courante/stagnante, durée d'inondation). Elle comprend 12 types pour les SDAGE, fortement inspirés de ceux développés pour l'évaluation des politiques publiques [10] et 28 types pour les SAGE, pour le moment encore trop proche des démarches par type d'habitat, la dimension fonctionnelle étant encore peu intégrée [8]. 
Par exemple, le projet de SDAGE du Bassin Loire Bretagne recense pour l'instant 320 zones humides. Il utilise soit la typologie du tableau 2 (voir en annexe), soit la typologie simplifiée suivante :

- estuaire et baie

— marais côtiers ou saumâtres, lagunes

- zones humides fluviales

- massifs riches en tourbières ou autres petites zones humides

- zones humides de prairie intérieure

\section{Exemples locaux}

Afin d'évaluer l'évolution de milieux fragiles de nombreux systèmes typologiques détaillés ont été décrits pour disposer d'inventaires, de cartes et de connaissances en général.

\subsection{Fonctionnement des zones humides}

L'effet lisière des zones humides caractérisées par des gradients et un continuum spatio-temporels, confère à ces zones des propriétés et un fonctionnement bien particuliers :

- un régime hydraulique marqué par la présence permanente ou intermittente de l'eau;

- des rôles multiples dans les cycles biogéochimiques;

- une forte productivité primaire: les zones humides sont parmi les milieux les plus productifs en matière organique de la planète; si la matière produite n'est pas directement accessible à l'homme, elle finira en grande partie par rejoindre les écosystèmes terrestres ou aquatiques : elle profitera alors à de multiples espèces végétales et animales dont certaines sont exploitées par l'économie humaine (pêche, chasse, agriculture, forêt,...)

- une diversité biologique remarquable: les zones humides, comme toutes les lisières, sont des lieux d'échanges écologiques, des zones à diversité spécifique élevée. Ainsi, un tiers des espèces rares ou menacées de notre pays sont inféodées aux zones humides.

Enfin, les zones humides remplissent divers rôles ou assurent de multiples fonctions. Ces fonctions sont difficilement perceptibles à l'échelle locale.

Ce n'est qu'au travers de bilans, souvent négatifs, à l'échelle d'un bassin versant par exemple, et la destruction des zones humides, que l'on peut mesurer combien ces fonctions sont indispensables à l'activité humaine. (tableau 1)

- Du point de vue de la ressource en eau, on sait par exemple que les zones humides assurent un rôle régulateur entre les eaux de surface et les nappes : stockage souterrain et superficiel, restitution progressive des volumes retenus, régulation climatique par le jeu de l'évaporation et l'évapotranspiration.

- Du point de vue de la qualité de l'eau, la végétation et les micro-organismes contribuent à la qualité de l'eau par ralentissement des écoulements, piégeage de matière en suspension et sédimentation, stabilisation de certains corps chimiques, consommation de nutriments, dénitrification. Des systèmes d'épuration s'inspirent d'ailleurs de ces processus naturels (lagunage) ou même utilisent directement des zones humides naturelles pour parfaire l'épuration des eaux résiduaires.

- Elles constituent un potentiel économique certain :

- la matière organique issue des zones humides permet le développement de micro-organismes dont se nourrissent de nombreuses espèces;

- les zones humides bordant les cours d'eau constituent des espaces essentiels pour la vie des poissons. Le brochet se reproduit par exemple dans les prairies inondées, d'autres poissons dans les bras annexes ou bras morts des cours d'eau. Des oiseaux migrateurs ou sédentaires s'abritent ou se reproduisent dans les mêmes milieux. Ces espèces font

\begin{tabular}{|c|c|}
\hline Fonctions & Conséquence après destruction \\
\hline $\begin{array}{l}\text { Action tampon vis-à-vis des crues, } \\
\text { retardement de la propagation des flux. }\end{array}$ & Inondation \\
\hline Stockage des eaux et recharge des nappes. & Sécheresse \\
\hline $\begin{array}{l}\text { Stockage des effluents naturels ou artificiels } \\
\text { et épuration des eaux polluées. }\end{array}$ & Augmentation des taux de pollution \\
\hline Régulation des cycles hydrologiques et chimiques. & $\begin{array}{l}\text { Phénomènes d'euthrophie/dystrophie, } \\
\text { problèmes de qualité des eaux }\end{array}$ \\
\hline Stabilisation des sédiments, protection des rivages/tempêtes. & Erosion des côtes et berges \\
\hline $\begin{array}{l}\text { Zones d'alimentation et de reproduction } \\
\text { de nombreuses espèces. }\end{array}$ & Diminution des pêches \\
\hline $\begin{array}{l}\text { Habitats et refuges pour une grande variété } \\
\text { d'espèces végétales et animales. }\end{array}$ & $\begin{array}{l}\text { Extinction d'espèces et diminution } \\
\text { de la diversité biologique }\end{array}$ \\
\hline
\end{tabular}

Tableau 1 - Conséquences de la destruction de zones humides. 
l'objet d'une exploitation plus ou moins extensive par la chasse et la pêche : ces espèces sauvages qui sont nées ou se sont nourries à un endroit seront souvent chassées en d'autres lieux [12].

Les zones humides constituent donc des espaces de grand intêrêt patrimonial. Elles assurent en outre diverses fonctions favorables aux activités humaines. Elles constituent bien des infrastructures naturelles $[8,12,13]$, qui au même titre que les infrastructures mises en place par l'homme, contribuent au maintien et au développement de ses activités.

Ainsi, les éléments qui font l'intérêt des zones humides acquièrent-ils une valeur collective de plus en plus élevée, parfois même potentiellement plus forte que la valeur d'usage. D'ou la nécessaire prise en compte par la collectivité pour assurer la préservation et l'entretien de l'existant et la reconquête des milieux dégradés. [12]

Les interrelations écosystèmes fluviaux - zones humides

Les connexions latérales et verticales, inscrites comme les connexions longitudinales, dans la dynamique des écosystèmes fluviaux, obligent à replacer les fleuves dans le contexte de leur environnement terrestre. Les interfaces ou écotones situées entre les fleuves et leurs plaines inondables ont une très grande importance, en particulier au niveau de la végétation riveraine (ripisylve et forêt alluviale); un rôle fondamental est également joué par les expansions et les contractions saisonnières des zones humides autour des cours d'eau en réponse aux régimes de précipitation, de la topographie locale et de la caractéristique des sols [14]. De même, des annexes hydrauliques déconnectées pendant de longs mois du chenal principal de grands fleuves «incisés», se comportent comme des zones humides pourtant situées dans le lit mineur.

\subsection{Les causes de la régression des zones humides}

- Les occidentaux ont opté pour la mise en valeur des zones humides par leur assèchement : dans les pays orientaux, les zones humides ont été généralement conservées. Les rôles et les fonctions de ces milieux auraient dû retenir notre attention plus tôt. [15]

- La plupart des opérations d'aménagement foncier se soldent par la mise en culture des fonds de vallée, le comblement des mares ou le redressement des rivières.

Il n'est donc pas étonnant de voir que les indicateurs physiques ou biologiques montrent une régression ou une dégradation des zones humides. [12]

- Les causes de dégradation ou de disparition des zones humides sont liées à l'activité humaine. Aux effets directs, défrichement et assainissement, s'ajoutent maintenant des effets indirects : abaissement des lignes d'eau des rivières et des nappes alluviales, rejet d'eau polluée, endiguement, pisciculture, populiculture. Par ailleurs, d'autres espaces sont abandonnés : les activités traditionnelles qui en assuraient l'entretien sont en très forte régression ou n'existent plus : pâturage extensif, récolte du bois et des productions végétales...

- Les variations climatiques actuelles peuvent accélérer la dégradation des zones humides en favorisant la croissance des végétaux ligneux contribuant au boisement des marais.

- En définitive, seules progressent les surfaces en eau stagnante résultant de la création de plans d'eau de loisir ou de l'abandon des ballastières : peu de ces milieux peuvent prétendre au statut de zone humide au sens de l'intérêt biologique [12].

- Cette régression, outre ses conséquences pour la flore et la faune, entraîne une dégradation lente des fonctions naturelles favorables aux activités humaines : régulation du régime hydraulique, autoépuration, action climatique. La construction d'équipements et les aménagements qui suppléaient ces rôles perdus, confirment cette dégradation (bassins tampon pour la régulation des débits, lagunes d'épuration, etc... voir tableau 1).

\section{LA RESTAURATION DES ZONES HUMIDES}

\subsection{Modalités mises en œuvre pour la protection et la préservation des zones humides}

\subsubsection{Principes à respecter pour la préservation des zones humides}

En ce qui concerne les zones humides d'intérêt majeur national ou local, il est préconisé de : (voir projet SDAGE de l'agence Loire Bretagne) [16]

- préserver la diversité des habitats et des espèces;

- préserver l'intégrité d'entités écologiques, veillant à ce que : - leur organisation et leur structure ne se dégradent pas (réseaux trophiques, cycles géochimiques,...),

- le réseau fonctionnel d'espace soit conservé ainsi que les superficies minimum viables du point de vue biologique,

- des corridors soient déterminés pour contrecarrer les effets de barrières dus à la présence d'étendues inhospitalières pour la flore et la faune,

- sur les territoires très anthropisés, certaines entités écologiques soient maintenues pour préserver leurs richesses biologiques.

- conserver le système naturel de régulation quantitative et qualitative de la ressource en eau (rétention naturelle des crues, autoépuration, restitution à l'étiage d'une eau de qualité).

\section{$\frac{2.1 .2 \text { Quels outils pour préserver et protéger les zones }}{\text { humides? }}$}

La préservation des habitats et des espèces ainsi que celle de l'intégrité d'entités écologiques des zones humides peut être menée à bien par :

- un acte légal (création de réserve, arrêté de biotope), du domaine de l'Etat, qui ne s'applique qu'à des surfaces relativement limitées si on les compare à l'étendue des zones concernées. Cette protection est accompagnée d'un programme de gestion;

- l'acquisition puis la gestion, en particulier par les Conser- 
vatoires du patrimoine naturel, des sites, etc, sur des fonds régionaux ou départementaux; dans le cadre des programmes LIFE (fonds européens);

En Loire amont, non endiguée, là où le fonctionnement dynamique fluvial s'inscrit dans un espace de liberté, la meilleure préservation des zones humides de la plaine alluviale ainsi que des annexes hydrauliques à l'étiage, passe par l'acquisition de ces espaces; le fleuve peut alors divaguer librement et retravailler l'espace, avec la seule contrainte d'une gestion patrimoniale.

Sauf exception, ces deux premiers outils, s'appliquant à des espaces limités, ont surtout valeur d'exemple ou de reconnaissance patrimoniale.

Les deux autres outils pouvant d'ailleurs aider à l'évolution des mentalités et des pratiques sont :

- la préservation par la voie d'une convention passée entre un propriétaire et les Organismes compétents qui peuvent être là encore les Conservatoires;

- la restauration ou la réhabilitation des zones humides dégradées, ou même la création de nouvelles zones humides. Un programme de gestion est là aussi généralement mis en œuvre.

2.1.3 Propositions d'actions d'accompagnement contribuant à la préservation des zones humides [16]

- Modalités de protection liées à l'agriculture :

En concertation avec le monde agricole, il serait souhaitable de mettre en oeuvre des mesures incitant à une gestion plus respectueuse des zones humides : plan de développement durable, mesures agri-environnementales, OGAF, plan de boisement, plan de gestion.

Par ailleurs, il serait souhaitable de supprimer certaines aides publiques aux programmes qui peuvent compromettre l'équilibre biologique des zones humides.

- Modalités de protection par rapport aux infrastructures :

Il devrait être interdit de pratiquer tous travaux ou prélèvements (en particulier, ceux qui dépasseraient les capacités de renouvellement de la ressource) susceptibles d'altérer gravement l'équilibre hydraulique et biologique des zones humides.

- Prise en compte des zones humides dans les documents d'urbanisme :

Il serait nécessaire d'identifier puis de transcrire les zones humides dans les documents d'urbanisme; les affouillements, les exhaussements du sol, les nouvelles constructions devraient y être réglementés. Par ailleurs, les boisements devraient être protégés par classement.

\subsection{Définition des actions qui caractérisent l'inter- vention humaine sur les hydrosystèmes et en particulier sur les zones humides.}

La multiplicité des termes utilisés pour caractériser les interventions humaines sur les hydrosystèmes, souvent pour réparer les dégats causés par le mauvais usage que l'homme a pu faire de la nature et de ses ressources, nous oblige à les définir, à travers certains concepts actuellement rassemblés sous le vocable général d'écologie de la restauration. [17]

La restauration consiste à favoriser le retour à l'état antérieur d'un écosystème dégradé, par le seul jeu d'un abandon ou d'un contrôle raisonné de l'action anthropique; la restauration impliquant que l'écosystème considéré, même s'il est dégradé, possède encore deux propriétés essentielles : être sur sa bonne trajectoire dynamique et présenter un bon niveau de résilience. [17]

La réhabilitation consiste à réparer les fonctions endommagées ou bloquées d'un écosystème, en ayant recours à des interventions plus lourdes, pour retrouver la trajectoire dynamique de l'écosystème et un bon niveau de résilience.

D'autres auteurs [18] proposent une autre définition de la restauration correspondant à des interventions visant un état idéal le plus proche de celui observé avant toute modification, un retour aux conditions «originelles» visant à :

— redonner à un écosystème endommagé, les conditions antérieures à la perturbation;

— rétablir ses attributs structurels et fonctionnels;

- relier ses caractéristiques physiques, chimiques et biologiques; on aurait donc un retour à un système en bonne santé, naturel et autorégulé, intégré dans le paysage naturel environnant [19].

Nous proposons ici de définir certains autres termes souvent utilisés :

l' «aménagement» consiste en une intervention sur les milieux naturels ou modifiés pour privilégier généralement un usage (épis pour la navigation, levées contre les crues, ...);

le «réaménagement» consiste à compléter, renforcer, transformer un aménagement afin de privilégier un usage ;

l'«entretien» consiste à maintenir une situation ou un état considéré comme bon ;

la création consiste à agir sur le milieu naturel ou anthropisé pour lui conférer le statut écosystémique de zone humide :

la préservation ou la protection consiste à placer les milieux sensibles à l'abri des dégradations -des mésusagesgrâce à des mesures conservatoires et une gestion appropriée ;

la «gestion» consiste à adapter les usages aux ressources (gestion patrimoniale d'une réserve naturelle, gestion de la ressource en eau, gestion de la navigation fluviale,...) ;

la gestion intégrée veut prendre en compte tous les usages...

le «génie écologique» correspond à l'application des sciences de l'ingénieur en écologie; il consiste à intervenir sur les milieux naturels ou modifiés en respectant le fonctionnement des écosystèmes pour aboutir à l'amélioration de la biodiversité

De la même façon que les zones humides ont pu être définies et caractérisées, il sera indispensable d'harmoniser la définition des interventions en matière de restauration, réhabilitation, création entre les scientifiques et les gestionnaires. 
En effet, peu de précautions sont prises quant à l'utilisation de ces termes, et sous une même rubrique se trouvent pêle-mêle des opérations aux objectifs d'ampleur et de niveau très différents [19].

Le terme restauration devrait être réservé aux interventions concernant des systèmes entiers. Or dans la plupart des cas, la délimitation et la nature des communautés ou des écosystèmes sont loin d'être cernés en raison, entre autres, de leur dynamique, de leurs changements cycliques ou directionnels sur des échelles de temps variables, même en l'absence de perturbations anthropiques [19].

\subsection{Essai d'approche typologique des interventions en restauration}

En fonction de leurs objectifs, nous pouvons identifier quatre catégories principales d'interventions en restauration :

La réhabilitation de sites très endommagés à la suite d'exploitations, de travaux d'aménagement etc... En France, la réhabilitation de carrières, gravières et sablières est un bon exemple de cette catégorie $[20,21,22]$.

La construction ex nihilo d'écosystèmes en compensation de destructions. Par exemple, aux Etats-Unis, la création de zones humides est réalisée par des bureaux d'études spécialisés en liaison avec des équipes scientifiques [19]. En France, au moins dans le cadre des programmes autoroutiers, on assiste à la création de mares dans un périmètre voisin de celles qui sont condamnées par la construction d'autoroutes [23]. Dans le même contexte, les bassins tampons évoluent souvent vers un lagunage paysager!

La conservation d'espèces, d'habitats, de la biodiversité, de sites protégés avec comme objectif le maintien d'un état donné ou le retour à un état antérieur jugé plus favorable du point de vue écologique. Dans la plupart des pays, les pratiques de la gestion patrimoniale appliquées aux réserves incluent depuis de longues années, sans les nommer, la restauration, la réhabilitation et la création. L'existence des réserves naturelles, l'achat ou l'établissement de conventions de gestion mis en oeuvre par les Conservatoires permettent de préserver les zones humides. L'évolution normale ou attendue des zones humides est d'aboutir à un stade d'atterrissement selon les schémas classiques. Seules les perturbations naturelles (inondations) à l'origine d'un rajeunissement continu des écosystèmes, la gestion «fixite» ou la restauration, permettent de conserver une gamme de milieux à des stades très variés. L'ouverture des milieux est dans ce cas maintenue par différentes méthodes patrimoniales : utilisation de races rustiques de chevaux, de bovins, le développement des activités pastorales $[24,25,26]$, les pratiques culturales aidées (mesures agri-environnementales, ...) etc.

Quelquefois, les mesures visant à favoriser certains groupes animaux tel l'avifaune, correspondent à un véritable aménagement de la nature, comme par exemple la création de clairières dans les roselières [26]

La restauration de bras morts ou d'annexes de cours d'eau sans objectif de réaménagement hydraulique mais visant à maintenir ou accentuer la biodiversité et à optimiser le fonctionnement des écosystèmes, concourt au même objectif.
On peut isoler une autre catégorie d'intervention dont l'objectif prioritaire est le rétablissement d'un bon écoulement des eaux, il s'agit :

- de la restauration des berges et de la ripisylve des petits cours d'eau rendue nécessaire par le manque d'intérêt des riverains et le désengagement de l'Etat pour l'entretien régulier et la gestion de la ripisylve. Cette restauration contribue dans le même temps à ce que l'écosystème retouve un meilleur fonctionnement et une plus grande biodiversité tout en s'inscrivant dans une démarche paysagère. Ces interventions peuvent donc être considérées comme une gestion patrimoniale incluant le génie écologique, le génie végétal, l'entretien régulier $[27,28,29,30,31]$.

- d'un réaménagement qui veut retrouver les conditions antérieures de l'aménagement du lit et dont l'objectif prioritaire est la sécurité des personnes en cas de crue (exemple de la Loire endiguée), mais qui veut prendre également en compte les préoccupations de restauration de la biodiversité et du meilleur fonctionnement des écosystèmes (Plan Loire Grandeur Nature, Etudes CNR sur le Rhône) [32 à 36], [37].

\subsection{Exemple de restauration de zones humides rive- raines de la Loire}

Les objectifs du plan Loire visent à relever la ligne d'eau à l'étiage et à rétablir l'hydraulicité normale de la Loire en intervenant en priorité sur les zones nouvellement atterries et fortement végétalisées, en respectant la biodiversité des écosystèmes.

Le plan Loire vise également à restaurer, par exemple dans leur fonction de frayère, les annexes hydrauliques, bras morts qui sont longuement déconnectés du lit vif incisé. La restauration de ces annexes peut avoir un objectif plus global qui est la restitution d'un milieu naturel écologiquement riche. L'intérêt n'est pas obligatoirement de favoriser la reproduction d'une seule espèce piscicole.

Une étude environnementale établissant l'état des lieux est indispensable pour proposer et valider les travaux à mener sur le site. Un certain nombre de données mésologiques et écologiques sont recueillies : estimation de la cote du fond de la boire, calcul en fonction des débits du creusement de la boire de manière à favoriser la reproduction du plus grand nombre d'espèces de poissons, topographie et communautés végétales, faune pisciaire, invertébrés benthiques, potentialités pour les vertébrés non poissons.

Ces données vont permettre de décider, en fonction des contraintes environnementales, de l'emprise optimale des travaux de restauration, ainsi que des méthodes pour réaliser cette opération.

Pour évaluer l'impact des travaux sur ces facteurs mésologiques et écologiques, le devenir des habitats floristiques et faunistiques ainsi que celui des espèces sensibles doit faire absolument l'objet d'un suivi. Ce suivi est particulièrement important dans un chantier expérimental dont les résultats doivent contribuer à l'élaboration d'un guide de restauration et de réhabilitation. Le grand nombre de familles d'invertébrés benthiques récoltées dans les boires montre l'importance écologique de ces annexes hydrau- 
liques. Le comblement naturel de ces zones engendrerait la disparition de cette richesse faunistique.

L'étude environnementale du chantier de restauration des «frayères» du bois chétif (La Chapelle/Loire-Chantier expérimental Loire moyenne) montre :

- une très grande différence de faune entre les prélèvements effectués dans le lit vif et ceux effectués dans chacun des deux systèmes hydrauliques annexes étudiés, différemment connectés avec le lit vif : on voit qu'il faut bien conserver la diversité des annexes hydrauliques.

- l'importance prépondérante des zones de bordure du lit mineur ou des boires qui apparaissent comme l'un des éléments majeurs des écosystèmes aquatiques qu'il faut préserver lors de la réalisation de travaux de restauration $[35,36]$.

Les études environnementales liées aux programmes de restauration sont souvent l'occasion de constater la faiblesse de nos connaissances fondamentales, de toute façon fragmentaires, relatives au fonctionnement dynamique des hydrosystèmes à tous les niveaux d'approche (global à l'échelle du bassin et des écozones, des unités fonctionnelles, au niveau habitationnel ou des microhabitats). Par exemple, sont mal établis, la caractérisation des habitats et leur dynamique (relations entre paramètres mésologiques et communautés floristiques mais surtout faunistiques; les successions temporelles et spatiales des communautés, l'harmo-

\section{BIBLIOGRAPHIE}

[1] Jouanin C. (1973) - Le projet MAR. Courrier de la Nature,27,1 $110-21$

[2] Barnaud G. (1990) - Synthèse bibliographique des typologies «zones humides : application aux Réserves Naturelles de France. Secrétariat d'Etat à l'Environnement, DPN. Laboratoire d'Evolution des Systèmes Naturels et Modifiés - MNHN. Rapport inédit, $39 \mathrm{pp}$ et annexes.

[3] Barnaud G. (1994) - Définition, typologie et caractéristiques de écosystèmes «zones humides». Stage «Connaissance et gestion des zones humides» - AFIE - Agence de l'eau Seine Normandie 20 p.

[4] Hansen A.J, F. di Castri, and P.G. Risser. (1988b) - A new SCOPE project. Ecotones in a changing environment: the theory and managment of landscape boundaries. Biol. Inernational, Special issue, 17, 137-163.

[5] Barnaud G. (1991) - Qu'est-ce qu'une «zone humide» Compte rendu des avis d'experts. Définitions scientifiques et juridiques. Laboratoire d'Evolution des Systèmes Naturels et Modifiés MNHN. Rapport inédit. $10 p$.

[6] Servan J. (1980) - Typologie, bilan, problématiques des espaces natutrels. Ministère de l'Environnement - DQV - MNHN, Rapport inédit, $91 \mathrm{p}$.

[7] Servan J., Blandin F. \& Loiseau J.M. (1982) - Typologie des milieux naturels. 3 volumes. Ministère de l'Environnement MNHN. Rapport inédit.

[8] Barnaud G. (1996) - Connaitre, protéger et restaurer les zones humides. Zones humides. $9 p$ et annexes.

[9] Lierdeman E. \& Mermet L. (1992) - Faisabilité d'un observatoire des zones humides. 3 volumes. Ministère de l'Environnement DPN, AIDA et SRPN. Rapport inédit.

[10] Mermet L, Cattan A, Jubault P. \& Lierdeman E. (1993) - Evaluation des politiques publiques en matière de zones humides. ASCA - Commissariat Général au Plan. Rapport de 1ère phase, 95 p. nisation des déterminations des espèces notamment entre larve et imago, l'étendue en terme de surface ou de linéaire occupée par les habitats et les communautés associées, la redistribution de la mosaïque des habitats après un événement hydraulique ou une intervention anthropique).

\section{CONCLUSION}

Tant que nos connaissances du fonctionnement des hydrosystèmes ne seront pas plus solides, il restera difficile de proposer aux gestionnaires des zones humides de vrais indicateurs de la qualité du milieu pour définir avec certitude les interventions en restauration-réhabilitation et création.

La rationalisation attendue de la restauration des zones humides doit passer par un approfondissement et une harmonisation de la recherche fondamentale ainsi que par le renforcement de la recherche appliquée. Cette rationalisation doit également passer par une véritable concertation entre les gestionnaires des zones humides et des cours d'eau : les ingénieurs et techniciens, les naturalistes et les scientifiques.

Enfin, avant toute restauration, il faudrait pouvoir apprécier et surtout maîtriser les pollutions d'origine anthropique ponctuelles ou diffuses qui détériorent gravement le fonctionnement des hydrosystèmes.

[11] Barnaud G . \& Comolet-Tirman J. (1995) - Correspondance entre des typologies zones humides et rivières. Agences de l'eau Rhin-Meuse et Rhône-méditerranée-Corse/Corine-Biotope/Ramsar. Lab. Evol. Syst. Natur. et Modifiés et Secrétariat de la Faune et de la flore, $13 \mathrm{p}$.

[11bis] Barnaud G. (1995) - Typologie nationale des zones humides applicables aux SDAGE et aux SAGE. Lab. Evol. Syst. Natur. et Modifiés - MNHN, Minsitère de l'Environnement DPN - DE, $12 \mathrm{p}$.

[12] Allion Y. (1996) - Les zones humides . Rapport pour la Conférence Régionale de l'Environnement - Région Centre, 9 p et annexes.

[13] Leynaud G. (1994) - Les zones humides. Les entretiens de Ségur. p $9-10$

[14] Naiman R.J. \& Décamps H. (1990) - The ecology and management of aquatic-terrestrial ecotones. Unesco. Paris.

[15] Lefeuvre J.C. (1994) - Les zones humides. Les Entretiens de Ségur. Ministère de l'Environnement, p. 6-8.

[16] Agence de l'eau Loire Bretagne (1995). Projet de schéma directeur d'aménagement et de gestion des eaux du Bassin Loire Bretagne - Dossier

[17] Le Floc'h E. \& Aronso J. (1994) - Ecologie de la restauration réponses à la dégradation des écosystèmes et à la fragmentation des paysages. Concepts, vocabulaire et applications. Natures Sciences - Sociétés., hors série.

[18] CAIRN J, JR (1988b) - Restoration ecology: the new frontier, 211 , in Cairn J. (ed) Rehabiliting damaged ecosystems, Boca Raton, Florida, CRC Press Inc.

[19] BARNAUD G (1995) - A l'interface de la pratique et de la théorie: l'écologie de la restauration. Natures - Sciences -Sociétés. hors série, $17 \mathrm{p}$.

[20] Frochot B \& Godread V. (1995) - Intérêt écologique des carrières, terrils et mines. Natures, Sciences, Sociétés, hors série. 
[21] BACCHI M. (1993) - Le réaménagement des carrières en eau. Eléments de connaissance et optimisation de la remise en état. DDAF du Loiret, IMACOF, Univ. Tours, 58p.

[22] Allion Y. (1994) Réhabilitation écologique et paysagère d'un site d'extraction de matériaux en lit majeur. Inst. Ecol. Appl. communication personnelle.

[23] BorTe F. (1996) - Université de Tours, CESA, communication personnelle.

[24] BAZIN P. (1996) - Espaces Naturels de France, Loire-Nature, communication personnelle.

[25] Lecomte T., Nicaise L., Leneveu C. \& Valot E. (1995) - Gestion écologique par le paturage: l'expérience des réserves naturelles. ATEN, 76p.

[26] Trotignon J \& Williams T. (1990) - Favoriser la vie des étangs. Ministère de l'Environnement, 67p.

[27] LACHAT B. (1994) - Guide de protection des berges de cours d'eau en techniques végétales. Ministère de l'Environnement, $143 \mathrm{p}$.

[28] LePETIT J. \& Gross F. ( 1994) - L'entretien régulier des rivières. Agence de l'eau Adour-Garonne, Rivière-Environnement, $89 \mathrm{p}$.

[29] Verniers G. (1995) - Aménagement écologique des berges des cours d'eau. Presse Univ. Namur, 8Op.

[30] MiChelor J.L, (1995) - Gestion patrimoniale des milieux naturels fluviaux. Rev. Nat. de France-ATEN, 67p.
[31] FLÜSSE UND B $\mathrm{A} C H E$. Erhalten, entwickeln, gestalten (1989) Wasserwirtschaft in Bayern oberote baubehorde bayern staatsminister, 164p.

[32] Dagorne D. \& BACCHI M. (1995) - Etude environnementale et cahier des charges du chantier expérimental d'Oudon. DIREN Pays de Loire, SMN, IMACOF-Université de Tours. 146p.

[33] NiCOLET S., BERTON J.P.\& BACCHI M. - Evolution topographique et recolonisation floristique du site de St Genouph après restauration du lit de la Loire. IMACOF, Univ. Tours, 57p. et annexes.

[34] RicOU G. \& BACCHI M. (1995] - Etude environnementale avant restauration du lit de la Loire entre la Charité sur Loire et Mesves sur Loire. DDE Nièvre, IMACOF - Université de Tours, $145 p$.

[35] BACCHI M. \& BERTON J.P. (1996) - Etude environnementale du site de Bréhémont avant restauration du lit de la Loire - Chantier expérimental Loire moyenne - Plan Loire. DDE d'Indre et Loire, IMACOF, Université de Tours, 83p.

[36] BACChI M., Berton J.P. \& Cornier T. (1996) - Etude environnementale du site de la Chapelle /Loire et des iles Jean Le Laid et proposition de restauration d'une annexe hydraulique (frayère du bois chétif) Chantier expérimental Plan Loire, DDE d'Indre et loire, IMACOF.96p.

[37] Henry C.P. \& Amoros C. (1995) - Restoration ecology of riverine wetlands. II. An exemple in a former channel of the Rhone River. Environmental managment, 19, 903-13.

\begin{tabular}{|c|c|c|c|c|c|}
\hline \multicolumn{3}{|c|}{ Dominantes } & \multirow[t]{2}{*}{ TYPES MAJEURS } & \multirow{2}{*}{ SDAGE } & \multirow[t]{2}{*}{ SAGE } \\
\hline Salinité & Régime & hydrique & & & \\
\hline \multirow[t]{21}{*}{ Eau douce } & \multirow[t]{7}{*}{ Eau courante } & $\begin{array}{l}\text { Inondée de } \\
\text { manière }\end{array}$ & \multirow[t]{7}{*}{ FLUVIALES } & \multirow{4}{*}{$\begin{array}{l}\text { Zones humides des cours } \\
\text { d'eau et bordures boisées } \\
\text { Plaines humides mixtes liées } \\
\text { aux cours d'eau }\end{array}$} & $\begin{array}{l}\text { Boisée } \\
\text { (ripisylve, fourrés } \\
\text { alluviaux) }\end{array}$ \\
\hline & & $\begin{array}{l}\text {-permanente } \\
\text {-saisonnière }\end{array}$ & & & $\begin{array}{l}\text { Herbacte } \\
\text { (prairies inondables) }\end{array}$ \\
\hline & & & & & $\begin{array}{l}\text { Palustre } \\
\text { (roseličre, cariçaie) }\end{array}$ \\
\hline & & & & & Végétation submergée \\
\hline & & -permanente & & \multirow{3}{*}{$\begin{array}{l}\text { Zones humides de } \\
\text { montagnes, collines et } \\
\text { plateaux }\end{array}$} & $\begin{array}{l}\text { Marais d'altitude } \\
\text { (source, combe à neige) }\end{array}$ \\
\hline & & - scisonniere & & & Tourbières \\
\hline & & & & & $\begin{array}{l}\text { ZH de bas-fond en tête } \\
\text { de bassin }\end{array}$ \\
\hline & \multirow{14}{*}{$\begin{array}{l}\text { Eau } \\
\text { stagnante }\end{array}$} & - temporaire & \multirow{4}{*}{$\begin{array}{l}\text { LACUSTRES } \\
\text { (Lac-Etang) }\end{array}$} & Régions d'étangs & Boisée \\
\hline & & $\begin{array}{l}\text {-saisonnière } \\
\text {-permanente }\end{array}$ & & \multirow[t]{3}{*}{ Bordures de lacs } & $\begin{array}{l}\text { Herbacée } \\
\text { (roselières, prairies } \\
\text { inondabies) }\end{array}$ \\
\hline & & & & & $\begin{array}{l}\text { Palustre } \\
\text { (roselière, cariçaie) }\end{array}$ \\
\hline & & & & & Végétation submergée \\
\hline & & - temporaire & \multirow{2}{*}{$\begin{array}{l}\text { MARAIS- } \\
\text { MARÉCAGES }\end{array}$} & \multirow{2}{*}{$\begin{array}{l}\text { Marais et landes humides de } \\
\text { plaine }\end{array}$} & Landes humides \\
\hline & & -permanente & & & \\
\hline & & $\begin{array}{l}\text { - permanente } \\
\text { - saisonnière }\end{array}$ & \multirow[t]{2}{*}{$\begin{array}{l}\text { [ZONES HUMDES } \\
\text { PONCTUEUES\} }\end{array}$} & $\begin{array}{l}\text { [Zones hurrides liées à un plan d'eau } \\
\text { ponctuel] }\end{array}$ & Petits lacs, Mares... \\
\hline & & & & [Prés-salés continentaux] & $\begin{array}{l}\text { Prés-salés } \\
\text { continentaux }\end{array}$ \\
\hline & & - Lemporaire & \multirow{2}{*}{$\begin{array}{l}\text { ZONES HUMIDES } \\
\text { AMÉNAGEESEN } \\
\text { EAUDOUCE }\end{array}$} & \multirow[t]{2}{*}{ Marais agricoles aménagés } & Rizières \\
\hline & & - saisonnière & & & $\begin{array}{l}\text { Prairies amendées } \\
\text { Peupleraies }\end{array}$ \\
\hline & & -permanente & & \multirow{3}{*}{$\begin{array}{l}\text { Zones humides aménagées } \\
\text { diverses }\end{array}$} & Résetvoirs-Barrages \\
\hline & & - temporaire & & & Carrières en cau \\
\hline & & & & & Lagunages \\
\hline & & & 7 TYPES MANEURS & 12 Types/SDAGE & 28 Types/SAGE \\
\hline
\end{tabular}

Tableau 2 - Typologie des zones humides hors eau salée-saumâtre. 


\section{Discussions au cours de la séance de la matinée du 13 juin}

A propos de la communication de M. Poitrineau concernant «L'Histoire de la Loire de la guerre de cent ans à nos jours».

La première question, soulevée par M. Roussel (Directeur adjoint de l'eau, Ministère de l'Environnement), porte sur le rôle de carrefour entre l'amont et l'aval joué par la ville d'Orléans sur la Loire. M. Poitrineau a ainsi ajouté que sur le Rhône, la ville de Lyon avait un rôle identique.

M. Roussel évoque ensuite l'utilisation et l'organisation collective de gestion, spécifiques à la Loire. En effet, la Loire a pour particularité de connaître une culture dite d'un "peuple d'eau". La vie s'est organisée autour du fleuve et en fonction de ses caprices.

En ce qui concerne l'utilisation du fleuve, M. Hospital insiste sur le fait qu'il est nécessaire d'être prudent. Par exemple, la navigation a connu une vitesse de développement certaine mais aussi un déclin rapide avec la disparition du profit qui a conduit à son abandon. Et il nous reste aujourd'hui un fleuve corseté. Que restera-t-il du fleuve dans 25 ou 30 ans?

Enfin, M. Cendrier (Commissaire - enquêteur), s'est interrogé sur le rôle des levées à leur origine. N'avaient-elles pas pour rôle principal de rassembler les eaux en période d'étiage, beaucoup plus que de protéger contre les crues ? M. Poitrineau fait remarquer qu'il y a eu une politique de "levées continues". En effet, le système de levées s'est mis en place progressivement et par à-coups, autant pour protéger les populations, que pour favoriser la navigation.

A propos de la communication de MM. Dauge, Bidault et Ayrault concernant «Le Patrimoine Ligérien».

$M^{\text {me }}$ Beaufils (Maire de Saint Pierre des Corps) démontre à juste titre que pour traiter correctement le patrimoine, chacun a besoin «d'aide» architecturale, paysagère... La perte du CAUE pour l'Indre et Loire a des conséquences douloureuses. Cette aide doit être recréée pour la réhabilitation de l'existant comme pour toute nouvelle réalisation. Selon M. Dauge, il serait nécessaire de mettre de l'argent dans des études paysagères.

$M$. Cazenave (SHF) fait alors constater qu'il nous manque en France une certaine vertu de continuité. On construit beaucoup, mais on n'entretient plus. Et la restauration demande un effort énorme. MM. Dauge et Bidault insistent sur le fait qu'à un usage est associé une gestion spécifique.

Pour conclure, M. Dambre (MISE) rappelle, tout en adhérant tout à fait à tout ce qui a été dit pour la sauvegarde du paysage ligérien, qui est très riche, varié et tout à fait remarquable, qu'il y a quelques siècles les villes s'étaient développées autour de la Loire, qui était alors l'axe économique principal, mais qu'avec l'arrivée du chemin de fer, le développement des villes s'en était ensuite écarté. Il lui paraît nécessaire de réorienter l'urbanisme des villes autour du fleuve, de façon à ce que celui-ci redevienne un axe de vie privilégié, tout en préservant les zones inondables qui doivent être réservées de préférence à des aménagements de loisirs. Il serait souhaitable de développer cet axe de réflexion dans les études d'urbanisme.
A propos de la communication de Mlle Babillot et de M. MARGAT concernant «Les Ressources et Utilisations de l'eau du Bassin de la Loire. Essai de comptabilité régionale de l'eau».

Suite à cette intervention, M. Duband (Président de division SHF) demande s'il n'est pas audacieux et dangereux de vouloir faire des bilans par mois compte tenu du temps de réponse des nappes et des incertitudes relatives aux prélèvements. A cela $M^{\text {lle }}$ Babillot rappelle que la comptabilité est faite sur une base trimestrielle et non mensuelle. M. Margat ajoute qu'ils sont conscients qu'il y a beaucoup d'estimations et qu'il faut faire attention aux marges d'erreurs. Ce sont les différences d'ordre de grandeur qui sont prises en compte et non les chiffres. En ce qui concerne les prélèvements, il y a une différence de perception entre les prélèvements pour l'agriculture et ceux pour l'eau potable (ces derniers étant mieux connus). La valeur des chiffres est donc différente.

Suite à cette discussion, M. Hospital invite l'assemblée à s'interroger sur la fiabilité des estimations concernant les prélèvements agricoles. En effet, les prélèvements agricoles sont mal comptabilisés du fait du manque de compteur. Et ceci est variable selon les régions. Mais il existe un espoir de pouvoir comptabiliser ces prélèvements correctement.

M. VILLEY (Ingénieur général MISE) intervint sur ce point pour rappeler qu'il est inutile de parler de gestion de la ressource «eau», si on ne mesure pas sérieusement les prélèvements, y compris agricoles. L'Agence de l'Eau Loire - Bretagne a demandé, il y a quelques années une politique volontariste de comptage de ces prélèvements et des compteurs ont été mis en place chez les exploitants.

Le dernier sujet de discussion porte sur la représentativité des chiffres en terme de consommation. Ainsi, il semble bon à M. Rousseau (France Nature Environnement) de rappeler que la consommation brute est définie comme le volume d'eau prélevé dans le milieu. Et la consommation nette est la différence entre la consommation brute et le volume qui retourne dans le milieu. M. Margat précise donc que la comptabilité prend en compte les consommations nettes.

Synthèse effectuée par Nina Dieu, secrétaire de séance

\section{Discussions au cours de la séance de l'après-midi du 13 juin}

\section{Discussions faisant suite à l'exposé de Mme Dacharry sur les grandes crues historiques de la Loire}

Le premier sujet de préoccupation de l'assemblée, abordé par M. Laperrouse (E.D.F), est la distinction entre les crues historiques de Haute-Loire, de Loire-moyenne et de la Loire aval, présentée par Mme Dacharry. En effet, cette distinction pourrait être due en partie à des aménagements successifs du lit, rendant historiques des crues qui antérieurement ne l'avaient pas été. Cependant cette distinction est permise, non pas par les divers aménagements réalisés sur ces différentes zones, mais uniquement grâce au fait que ces secteurs sont distincts par leurs morphologie, météorologie,... De plus, aux périodes considérées (XVIIİ̀me siècle, 1846, 1910), il n'existait pas d'aménagement 
majeur pouvant porter à conséquence sur les niveaux de crues. Ainsi, la crue de 1910 marqua aussi bien les secteurs de Loire aval que les secteurs de Haute-Loire.

M. Maurin demande ensuite la précision avec laquelle on peut espérer déterminer les débits des crues de 1846, 1856, 1866 , sachant que l'on connaît avec une bonne précision les hauteurs d'eau mais que les profils sont, eux, très mal déterminés.

A cela $\mathrm{M}^{\mathrm{me}}$ Dacharry rapporte que les modifications du lit mineur étaient, en effet, très importantes et qu'on avait des problèmes d'approximation des débits. Néanmoins, elle estime la précision sur les valeurs à $10 \%$ environ du débit. L'ordre de grandeur des crues de $1846,1856,1866$ étant de $7500 \mathrm{~m}^{3} / \mathrm{s}$, on peut donc retenir une valeur de référence de $8000 \mathrm{~m}^{3} / \mathrm{s}$.

En ce qui concerne, les protections contre ces crues exceptionnelles, l'assistance s'interroge sur les limites de cette protection vis-ầ-vis des différents types de crues. Ainsi, il semble bon de rappeler, comme l'a fait fort opportunément $\mathrm{M}^{\text {me }}$ Dacharry, que si les levées de Loire constituent une bonne protection contre les petites crues et les crues moyennes, elles ne protègent pas contre les très grandes crues et au contraire aggravent les dommages causés par celles-ci. Il est important, fait remarquer M. Dambre, de ne pas oublier que les levées de la Loire ne sont pas une protection absolue et que les zones inondables situées derrière les levées (comme à Tours) doivent être préservées car elles constituent pour ces crues des champs d'expansion possibles.

Les trois crues historiques de 1846,1856 et 1866 représentent chacune un événement unique, aux conditions de genèse particulières, étroitement liées à l'hydrométrie. Ainsi, crues de rétérence et phénomènes météorologiques jouent un rôle dans l'annonce des crues. Reste à savoir dans quelle mesure l'étude des phénomènes locaux est indispensable ? $M^{\text {me }}$ Dacharry montre que ces études locales étaient nécessaires car un phénomène météorologique important, même à $3 \mathrm{~km}$ près, peut changer la course des événements. En réalité, les scénarios pour connaître la genèse de ces crues sont nombreux (influence de l'homme, météorologie ...). Mais la prise en considération de ces crues de référence prend tout son sens dans la connaissance des champs d'expansion de crues.

On arrive alors logiquement à parler de l'influence de l'absence de végétalisation sur l'évaluation des crues du XIX ${ }^{\text {ène }}$ siècle en comparaison à la situation de la Loire actuelle dont le lit est fortement végétalisé, fait remarquer à juste titre $M$. Coste. Il est vrai que de ce point de vue, beatucoup de travail reste à faire pour permettre à la Loire de retrouver son "visage d'antan"; ce grand nettoyage devant être regroupé avec d'autres actions déjà amorcées par l'arrêt d'extraction de granulats dans le lit du fleuve. Une bonne appréhension des différentes causes de cette abondante végétalisation du lit majeur du fleuve est nécessaire avant la réalisation de tels travaux.

Enfin, M. Gazowski apporte quelques précisions concernant la connaissance acquise des débits de crue au milieu du XIX ${ }^{\text {ìme }}$ siècle qui, pour sa part, sont d'une précision analogue à celle d'aujourd'hui. Les raisons en sont :

- la mesure des débits se fáisait avec les mêmes techniques qu'aujourd'hui (au moulinet type Woltmann dont un exemplaire se trouve au Musée de Munich),

- les courbes de tarage de l'époque présentaient une grande stabilité due à un bon entretien du lit du fleuve et à l'absence quasi totale de prélèvement de granulats,

- malgré une technologie de mesure de débit plus performante, l'établissement des courbes de tarage est plus délicat aujourd' hui en raison de l'évolution très rapide du lit.

\section{Discussions faisant suite à l'exposé de D. Duband sur la genèse des crues dans le bassin de la Loire.}

La première discussion porte sur la protection contre les crues faibles et moyennes. $M$. Picard signale l'observation faite concernant ce type de protection qui apparaît satisfaisant lorsque la zone protégée a une occupation qui ne subit pas de commages tróp graves comme en rive gauche de la Loire dans la région de Sancerre.

Le second point de discussion porte sur l'état actuel des connaissances et de leur rôle dans la prise de décision ainsi que sur les risques encourus en amont du bec d'Allier pour une crue de débit supérieur à celle du siècle dernier. Aujourd'hui, on a tendance à plafonner dans les connaissances des crues au XVII ${ }^{\text {mme }}$ siècle; mais maintenant on se permet d'extrapoler les résultats, de faire des prévisions.

M. Duband évoque l'exemple envisagé d'une crue millénale entre Gien et Blois afin d'être le plus concret possible. Une telle crue constituerait un débit de $11000 \mathrm{~m}^{3} / \mathrm{s}$, ce qui est fort plausible. Cependant, en l'état actuel des connaissances et des ouvrages d'art, aucun barrage ne peut gérer ce type de crues. Pour des crues moyennes, il est possible d'établir des règles de gestion. La mise en oeuvre se fait par la gestion de barrages écrêteurs de crues. Leur fonction est valable pour des bassins versants de quelques dizaines de $\mathrm{km}^{2}$. Il est extrêmement important d'écrêter la pointe de crue mais tout le problème réside dans la détermination du lancement de la phase d'écrêtement. Dans le cas de crues de grande importance, seuls les champs d'inondation permettent de gérer au mieux ces événements. Quant aux crues locales, ce sont les plus difficiles à prévenir.

\section{저․ Discussions faisant suite à l'exposé de M. Camp'huis sur la stratégie d'écrêtement des crues en Loire moyenne}

La première question de $\mathrm{M}^{\mathrm{mc}}$ Beaufils attire l'attention de l'assemblée sur la façon de travailler de cette équipe de 3 partenaires (Agence de l'eau, E.P.A.L.A., Etat) pour prendre en compte l'évolution de l'ensemble du val entre 1856 et aujourd'hui, alors que le val doit être très différent de ce qu'il était en 1856.

En fait, une nouvelle topographie a été réalisée en 1995 ainsi qu'une modélisation mathématique en juin 1996. Ce val est inclus au P.O.S., constitué avec l'aide de l'IFEN pour les vals ruraux et à l'aide de photos aériennes au 1/25000 pour les autres zones du val.

Les vals sont très peu différents entre deux périodes. En effet, dans tous les cas, d'une part la vitesse d'entrée dans le val est faible et d'autre part, la vitesse lors des vidanges est importante, ce qu'il reste à vérifier par la modélisation.

M. Degardin attire ensuite l'attention des techniciens et des politiques sur la nécessité d'éviter l'illusion de la maitrise totale du risque, de la sécurité totale car elle n'existe pas. Le meilleur aménagement que peut faire l'ingénieur et le politique, n'est pas celui qui assure un seuil de protection de plus en plus élevé (cinquantennal, centennal, pluriannuel ...) mais celui qui assure la sécurité en cas de dépassement de la protection qui se produira inévitablement. D'autre part, une stratégie de gestion du risque doit comporter une part importante d'information des populations.

Une stratégie de prévention a été mise en place. Ainsi, on possède désormais le réseau C.R.I.S.T.A.L. qui constitue une cel- 
lule de prévention des crues efficace. Néanmoins, un problème demeure sur la prise de décision qui sera décisive lors de crues de débit de $10000 \mathrm{~m}^{3} / \mathrm{s}$. Tout est entre les mains du Préfet. En ce qui concerne les plans d'évacuation, ils sont surtout départementaux. Le plan municipal de secours d'Orléans, par exemple, est en cours d'élaboration. La pointe limite de débit décidant l'évacuation des populations est fixée à $6500 \mathrm{~m}^{3} / \mathrm{s}$.

M. Auverm intervient sur ce point afin de rappeler qu'une connaissance précise des processus d'inondation des vals est indispensable pour mettre au point les plans de secours. Ces connaissances sont aujourd'hui insuffisantes et nécessitent un travail de modélisation encore à entreprendre dans différents secteurs.

Melle Julian du conservatoire du Patrimoine Naturel de la Région Centre, aborde le problème de l'abandon des zones annexes de la Loire qui est essentiellement dû à l'enfoncement du lit et non à un abandon de l'entretien de la végétation du lit. Il s'agit donc là d'un problème beaucoup plus complexe, l'enfoncement du lit de la Loire se traduisant par l'abaissement de la ligne d'eau à l'étiage. Elle rappelle également que la diversité naturelle des rives et vals de la Loire est liée aux crues et inondations périodiques. Ainsi un patrimoine exceptionnel est présent à l'amont de Briare. On trouve là une forêt alluviale dont la richesse, la diversité et les spécificités sont dues aux crues. Il faut donc bien différencier crues et inondations : le champ d'expansion des eaux n'est alors pas le même. Pour conserver cette richesse, il est nécessaire de gérer également ces zones d'expansion de crue en tant que «réserve naturelle».

Un conseiller à la ville de Tours attire l'attention de l'assemblée sur les dégâts de plus en plus importants derrière les levées. Gérer au mieux l'urbanisation des vals et des coteaux devient un enjeu majeur. De plus, pour contrecarrer ces inondations, certains vals vont être désignés comme zone préférentielle pour l'expansion des crues. Une gestion du risque et une étude descriptive des dégâts sont à établir. Ces vals jouant un rôle très important dans l'écrêtement des crues exceptionnelles doivent être les partenaires de barrages écrêteurs de crues. Cependant, la reconquête du lit endigué par une restauration et un entretien pourrait permettre un amortissement des crues exceptionnelles. Désormais, seuls les responsables décideront de ce qu'il reste à entreprendre au sein de telles politiques.

Le dernier sujet de discussion porte sur la stratégie d'écrêtement entre le barrage du Veurdre et celui de Villerest sachant qu'il est souvent question de cette retenue lorsque l'on parle des problèmes de la Loire. Est-ce qu'une modélisation peut être une aide à la décision pour le gouvernement ? Quels sont les choix offerts au gouvernement à partir d'une modélisation ?

L'ouvrage d'écrêtage du Veurdre est un barrage sec. Il fonctionne par abaissement de vannes sectorielles à partir de 3000 $\mathrm{m}^{3} / \mathrm{s}$. Cet ouvrage ne se ferme jamais complètement et les poissons pourront toujours passer en temps de crue. Le volume utile du barrage du Veurdre est de 90 millions de $\mathrm{m}^{3}$. Le problème réside dans le choix du moment où il faudra abaisser les vannes afin d'écrêter la crue. Voici quelque temps, le débit limite d'abaissement des vannes est passé de $8500 \mathrm{~m}^{3} / \mathrm{s}$ à $4000 \mathrm{~m}^{3} / \mathrm{s}$.

Quant à l'influence d'une modélisation sur les décisions gouvernementales, elle permet d'illustrer l'impact d'une politique d'entretien et de restauration à différents niveaux. Cela a été démontré avec la modélisation de 1982 qui illustre l'impact d'une politique au niveau local montrant l'influence de la végétalisation sur l'écrêtement des crues et la formation d'atterrissement par défaut d'entretien.

\section{留 Discussions suite à l'exposé de M. Grassin sur l'évo- lution de la politique de prévention des inondations.}

L'intervention de $M$. Grassin amène une réflexion de $\mathrm{M}^{\text {me }}$ Beaufils. En effet, St-Pierre-des-Corps, dont elle est le Maire, est un secteur inondable et une intercommunalité entre les communes ayant le même problème, ne résoudrait pas tout d'après elle. Car une commune à une vie, des envies de développement propres à elle-même, mais les risques demeurent néanmoins. Les communes protégées par des digues doivent se réunir afin de réfléchir à des actions communes. En fait, il n'y a pas de solution sans débat public préalablement préparé par la diffusion d'une information pertinente aux responsables et aux populations (privés, industriels etc.) afin de faire évoluer les réflexions personnelles sur ces problèmes de gestion du risque. Depuis 1994, dans les discussions, on fait la part entre ce qui existe déjà et ce qui n'est pas encore en matière de risque car il est indispensable de prendre des mesures de protection sans faire augmenter le risque. Dans cette gestion du risque d'inondation, il faut laisser une zone d'expansion à la crue.

En dernier lieu, l'établissement d'une stratégie de gestion des crues permettra d'orienter les stratégies de protection des zones à forte vulnérabilité (comme St-Pierre-des-Corps par exemple). Ces stratégies permettront de mieux percevoir l'avenir, d'aider l'Etat à prendre les bonnes décisions et de choisir les politiques adéquates à la restauration du milieu naturel fluvial.

Faute de temps, les communications de M. Soquet et M. Dumay ne purent faire l'objet de discussions avec les membres de l'assemblée.

Synthèse réalisée par Armelle Sicart, secrétaire de séance

\section{Discussions au cours de la séance de la matinée du 14 juin}

\section{Discussions suite à la communication de M. Leroy «Comment concilier économie et écologie dans l'estuaire de la Loire» (communication présentée par M. Villey)}

M. Maurin, Président de la SHF demande à M. Villey de donner son opinion personnelle sur le sujet présenté au nom de $\mathrm{M}$. Leroy. M. Villey répond que M. Leroy sous-estime la décision prise par le ministre car il demeure une question non résolue : la Virgule de Barbeuf. En effet, cette zone localisée dans la partie sud de l'estuaire est vouée à disparaître car elle est en train de s'envaser. On risque de perdre un site d'une grande valeur écologique. Il se demande ce que l'on peut faire pour que cet endroit demeure en eau.

M. Pinte (EDF) demande quels sont les travaux prévus pour réduire l'importance du bouchon vaseux dans l'optique d'assurer une meilleure circulation des poissons migrateurs. La première chose à faire est de réduire les oscillations du bouchon vaseux. Il faut donc faire des travaux de déchenalisation à l'amont, et ce très vite. Cela va permettre le relèvement de la ligne d'eau. Mais il faut pour cela démontrer à la population que la navigation à l'amont de Nantes n'a plus aucun intérêt économique. 
M. Décamps s'inquiète du devenir du modèle élaboré. M. Villey précise qu'aucun financement n'a été prévu pour faire perdurer ce modèle car généralement il est rare qu'un tel outil continue à «vivre». Mais Nantes a une volonté contraire et veut utiliser ce modèle afin d'obtenir une cellule de mesures et vulgariser les résultats.

Discussions suite à la communication de MM. Mignot et Lefèvre sur la problématique des étiages

M. Rousseau (France nature Environnement) intervient à propos des conséquences induites par le barrage de Naussac. Selon la période d'hydraulicité (forte ou faible), l'impact du barrage est minime ou très important. Faire une moyenne, comme dans le document présenté à propos de Naussac, revient à masquer l'impact du barrage sur les débits, dans sa période d'utilisation réelle. Ce document, présenté par l'EPALA, n'est pas plus convainquant que le catalogue des avantages du soutien d'étiage pour le barrage de Chambonchard pour lequel on cherche une réelle analyse des inconvénients.

Discussions suite à la communication de M. l'Hermitte sur EDF et la Loire : 50 ans d'histoire commune

M. Masson demande ce qui va se passer pour le barrage de Maisons-Rouges. EDF appliquera les décisions gouvernementales, que l'effacement soit confirmé ou à l'inverse que le barrage soit maintenu. Il est toutefois nécessaire que la décision soit prise rapidement. Quant aux nouveau projets de micro-centrales, ils sont surtout à buts multiples (électricité, irrigation, eau potable).

M. Rousseat (France nature Environnement) contredit M. L'hermitte au sujet de la réussite des vidanges de barrages. Il estime que la vidange d'Eguzon sur la Creuse en 1987 est une véritable catastrophe. La matière solide transférée à l'aval a rempli complètement le lit de la rivière, à l'exception d'un chenal d'une quinzaine de mètres dans lequel coulait un fluide plus proche de la boue que de l'eau. Les poissons et la faune ont souffert et l'eau potable à l'aval a été polluée.

M. Villey témoigne de l'intéressante collaboration qui s'est instaurée avec EDF depuis quelques années. Il demande quelle est la position d'EDF vis-à-vis des ouvrages qui produisent peu d'électricité et qui ont un mauvais impact. En ce qui concerne les petites centrales hydrauliques, dans la mesure où les ouvrages existent, EDF essaie de diminuer leur impact et considère que l'on doit continuer à les développer.

iㅗㄹ Discussions suite à la communication de M. Dambre sur les extraction de matériaux dans le lit mineur et le lit majeur de la Loire et de ses affluents

M. Bethemont demande quelles sont les définitions retenues pour le lit mineur et le lit majeur. Les définitions prises en compte pour le cas présent sont les suivantes : 'le lit mineur est le lit mouillé en moyenne plus de 10 jours par an et le lit majeur représente les zones inondables moins le lit mineur.

M. Bethemont demande également quel est l'impact sur la qualité des eaux de ces extractions de sable jouant un rôle de filtre purificateur. M. Dambre répond qu'un abaissement de la ligne d'eau a été constaté mais que le sujet mériterait plus amples études.

Discussions suite à l'intervention de MM. Gougis et Steinbach sur le retour des poissons migrateurs

Compte tenu qu'EDF fait face à ses obligations en matière de franchissement des obstacles à la migration, M. Pinte aurait souhaité que le Conseil Supérieur de la Pêche montre les résultats de la migration à Poutès et tente de donner une explication de la diminution des effectifs. Poutès est équipé d'un ascenseur et le passage des poissons est filmé, ce qui permet un comptage précis. Ainsi, en 1988, une bonne répartition entre les saumons de printemps et d'automne a pu être observée. Le cycle du saumon dépend de plusieurs éléments qui doivent se conjuguer, sinon, cela peut conduire à l'extinction de l'espèce. Cette année, les chiffres sont encourageants mais ne permettent pas de conclusion hâtive.

\section{Discussions suite à la communication de MM. Berton et Bacchi sur la restauration des zones humides}

M. Dambre souligne que le rapport de juin 1994 de l'instance d'évaluation des politiques publiques en matière de zones humides a mis en évidence la dégradation continue de la plupart des zones humides en France. La moitié d'entre elles ont disparu au cours des trente dernières années. La réduction maîtrisée des extractions de matériaux dans le lit majeur est l'une des conditions nécessaires à la sauvegarde des zones humides.

Synthèse réalisée par Fabienne Ragot, secrétaire de séance 


\title{
Table ronde
}

\author{
Présidée par M. Guellec, Président du Comité de bassin Loire-Bretagne, \\ cette table ronde accueillait comme participants :
}

MM. Décamps, Doligé, Duband, Masson, Rousseau, Villey-Desmeserets

\begin{abstract}
M. Villey-Desmeserets, chargé de mission d'inspection spécialisée du ministère de l'Environnement, résume les points essentiels du congrès, qui n'est pas, à son sens «un colloque Loire de plus» comme il y en a tant, car son contenu est beaucoup plus complet, notamment par une large évocation de tous les aspects biologiques et par les exposés et discussions sur le patrimoine. Il espère qu'il y a progrès dans la prise en compte de cette dimension. Il note qu'on a longuement parlé des crues et inondations et qu'on a bien expliqué les mécanismes. Il reste à bien les faire comprendre à l'opinion. A propos des étiages et de leurs prévisions, il retient qu'il serait bien nécessaire d'avoir une comptabilité précise des prélèvements. Les problèmes liés aux extractions de granulats et leur règlementation ont été justement signalés. Il faut souligner également la remise en valeur des zones humides et un bon exposé sur les poissons migrateurs... en espérant à ce propos la mise en place d'un programme sérieux. Il demande si l'EPALA compte faire quelque chose en ce domaine.
\end{abstract}

M. Doligé, député, Président du Conseil Général du Loiret, présente brièvement le fonctionnement actuel de l'EPALA, qu'il préside, et souligne son intérêt pour le rôle de la Loire dans l'aménagement du territoire, et pour l'amélioration de la vie des poissons migrateurs.

Le Professeur Décamps, écologue, directeur de recherche au CNRS, souligne l'intérêt des recherches en écologie. Se posant la question «qu'est-ce qu'une belle rivière ?», il répond que c'est d'abord un cours d'eau qui vit, donc qui varie, qui évolue avec ses pulsations propres. Mais jusqu'où peut-on varier ? Il y a certes nécessité de protections, mais à condition de maintenir l'identité et l'intégrité du fleuve. D'où l'importance de la notion de «continuuum» ou de «connectivité» du fleuve. Comment restaurer les flux, et à quelles échelles ? Cela doit se traiter dans un esprit de discussion ouverte, de communication franche entre les usagers. Un beau fleuve est un «fleuve qui inspire»... aussi bien les poètes que les projets. Pour l'écologue, il ne suffit pas de connaitre «l'anatomie» mais aussi de bien comprendre la «physiologie», ce qui implique l'intelligence de restaurer et d'aménager.

M. Duband, Président de la division «Eau» de la SHF, pense qu'il faudrait revenir sur le problème des étiages, au moins aussi grave que celui des crues, et sur les questions relatives aux interactions entre eaux souterraines et eaux superficielles qui n'ont pu être traitées en raison de l'absence de M. Ausseur. Il souligne que la Loire peut être comparée à l'Ebre, fleuve espagnol qui a également gardé un caractère sauvage. Il insiste sur l'importance de la communication avec les riverains en ce qui concerne les événements exceptionnels.

M. Rousseau, au nom de «Loire vivante» et de «France Nature environnement» déplore les difficultés de communication en matière d'écologie; il estime que le colloque a été marqué par une volonté de discussions plus ouvertes, moins triomphalistes qu'en d'autres manifestations du même genre. Il regrette cependant qu'on n'ait pas eu le temps de parler du projet du Veurdre. Il estime que si les solutions envisagées pour les crues sont correctes, il y a lieu d'être plus réservé sur les soutiens d'étiage. Qu'en est-il des éléments en trace ? Et de la qualité des eaux ? La carte des nitrates est inquiétante, celle des phosphates aussi. Et le patrimoine s'appauvrit : le bocage du Perche est grignoté par l'aspect «grandes cultures» de la Beauce. Pour faire avancer les choses, il faudrait faire coopérer les mouvements associatifs et l'EPALA.

Le Président Guellec, partant de ces propos, estime qu'il y a lieu d'élaborer une politique d'ensemble concertée, le soin du fleuve ne pouvant être laissé entièrement ni aux associations d'écologistes, ni à EDF.

M. Masson, coordonnateur de l'eau à EDF, ainsi mis en cause, donne l'esprit du rôle d'EDF, qui est à la fois aménageur et gestionnaire de l'eau, plutôt gestionnaire actuellement car les aménagements sur la Loire sont terminés. L'objectif est de faire vivre chaque fleuve avec son caractère propre, même avec la présence de l'industrie, dont EDF. L'établissement est favorable à un large esprit de partenariat avec tous ceux qui ont en charge les activités et préoccupations riveraines.

Le Président Guellec conduit alors un dialogue avec la salle où s'expriment les aspirations de certains élus pour la défense de l'environnement, les regrets de na pas laisser assez de place aux sciences humaines, l'inquiétude devant les schémas directeurs d'urbanisme qui ne tiennent pas compte des crues (ce point devrait s'améliorer). M. Relosato, adjoint au maire de Givors, souhaite une participation des élus municipaux à tous les problèmes liés aux rivières, notamment en dynamique fluviale, en définition des champs d'expansion des crues...

A un participant qui souhaite voir la SHF prendre position sur la politique fluviale, le Président Maurin rappelle la mission de la SHF : «Favoriser le progrès des connaissances dans les domaines des sciences hydrotechniques (hydraulique, mécanique des fluides et les sciences qui s'y rattachent), mais aussi dans les domaines des sciences de la terre et de la nature (utilisation, répartition et protection de la ressource en eau, en quantité et en qualité)».

Dans ces domaines de l'hydrotechnique et de l'environnement, elle est le point de rencontre privilégié de scientifiques, industriels, ingénieurs et gestionnaires qu'elle rassemble au sein d'un Comité Scientifique et Technique.

En clôture, M. Cazenave, Président du Comité Scientifique et Technique de la SHF, rappelle la part grandissante de la biologie, de l'écologie et des sciences de l'environnement, sensible depuis une bonne dizaine d'années dans les travaux couronnés par le prix d'hydrologie de la SHF (prix Henri Milon) et de plus en plus dans la plupart des colloques ou congrès. Il se félicite d'avoir suggéré le sujet sur l'histoire de la Loire, de la guerre de cent ans à nos jours, l'exposé excellent du Professeur Poitrineau et le brillant tableau brossé par M. Dauge et ses collaborateurs ayant créé un esprit «patrimonial» qui a marqué un peu tout le colloque. Il se réjouit d'une bonne participation des élus à ce colloque, qui couronne l'effort en ce sens de la SHF qui naguère encore ne touchait que des scientifiques et des ingénieurs. 